\title{
Factors Predicting Weight Loss after "Sleeve Gastrectomy with Loop Duodenojejunal Bypass" Surgery for Obesity
}

\author{
Amar Vennapusa ${ }^{1, *}$, Ramakanth Bhargav Panchangam², Charita Kesara', Tejaswi Chivukula ${ }^{1}$ \\ 'Department of Bariatric and Metabolic Surgery, Dr. Amar Bariatric \& Metabolic Center, Hyderabad; '2Department of Endocrine and Metabolic Surgery, Endocare \\ Hospital, Vijayawada, India
}

Background: Laparoscopic sleeve gastrectomy with loop duodenojejunal bypass (SG LDJB) is a loop modification of biliopancreatic diversion with duodenal switch. The aim of this study was to analyze weight loss response and factors predicting weight loss outcomes after SG LDJB.

Methods: This was a retrospective study analyzing SG LDJB surgeries performed between May 2013 and December 2017 in 126 Indians suffering from obesity. The collected data was analyzed to understand weight loss efficacy and the factors predicting weight loss. Surgery was considered successful when percentage excess weight loss (\%EWL) was $\geq 50 \%$ or percentage total weight loss ( $\% \mathrm{TWL}$ ) was $\geq 25 \%$.

Results: \%EWL was $95.77 \%$ and $83.84 \%$ and \%TWL was $34.64 \%$ and $30.32 \%$ at the 1 -year and 3-year followup, respectively. $\%$ EWL $\geq 50 \%$ was $99.04 \%$ and $96.47 \%$ and $\%$ TWL $\geq 25 \%$ was $91.35 \%$ and $75.29 \%$ at the 1 -year and 3-year follow-up, respectively. Patient age and sex did not independently predict \%EWL or \%TWL. Preoperative body mass index (BMI), weight, and excess weight negatively predicted \%EWL and positively predicted $\%$ TWL at the 1-year and 3-year follow-up. Multiple regression analysis showed that these parameters were independent predictors of \%EWL and \%TWL at 1 year, while preoperative weight and excess weight also independently predicted \%EWL at the 3-year follow-up. In diabetic patients, \%EWL was significantly lower at the 3-year follow-up and \%TWL was significantly lower at the 1-year and 3-year follow-up.

Conclusion: SG LDJB was an effective bariatric surgery to treat obesity. Preoperative BMI, weight, excess weight, and the presence of diabetes were the significant factors associated with the weight loss outcomes.
Received May 18, 2020

Reviewed June 11, 2020

Accepted August 19, 2020

*Corresponding author

Amar Vennapusa

(iD)

https://orcid.org/0000-0001-7338-1922

Department of Bariatric and Metabolic Surgery, Dr. Amar Bariatric \& Metabolic Center, Plot No - 99, Ground Floor, Avis Hospital, Next to Chiranjeevi Blood Bank, Opposite to Metro Pillar C1588, Road No. 1, Jubilee Hills Check Post Road, Jubilee Hills, Hyderabad, Telangana 500033, India Tel: +91-96766-75646 E-mail:drVamar@gmail.com

Key words: Bariatric surgery, Obesity, Weight loss, Diabetes, Metabolic surgery

\section{INTRODUCTION}

Obesity is considered a serious health and socioeconomic problem worldwide. ${ }^{1}$ Obesity leads to the development of co-morbid conditions such as type 2 diabetes, hypertension, hyperlipidemia, and coronary artery disease. ${ }^{2}$ Lifestyle modifications such as diet and exercise are the basic treatment modalities to obviate obesity. Weight loss with lifestyle modifications can be unsuccessful in those with severe obesity, hormonal imbalances, metabolic disor- ders, or genetic problems. Pharmacotherapy has been found to be ineffective in the long run. ${ }^{1}$

Surgical intervention has proven to be superior compared to lifestyle modifications and medical treatment in dealing with patients suffering from severe obesity. ${ }^{3}$ Bariatric surgery is the most effective technique for reducing body fat. The physiologically induced metabolic changes, hormonal changes, alterations in intestinal microflora, and modifications in nerve signaling contribute to the loss of body fat after bariatric surgery. ${ }^{1}$

Copyright ( 92020 Korean Society for the Study of Obesity

(.) This is an Open Access article distributed under the terms of the Creative Commons Attribution Non-Commercial License (https://creativecommons.org/licenses/by-nc/4.0/) which permits unrestricted non-commercial use, distribution, and reproduction in any medium, provided the original work is properly cited. 
Laparoscopic biliopancreatic diversion with duodenal switch (BPD DS) is the most effective bariatric surgery for long-term weight loss and remission from diabetes. ${ }^{4}$ However, risk of severe malabsorption is very high in BPD DS since a significant length of the small intestine is bypassed. ${ }^{5}$ The gold standard bariatric surgery, laparoscopic Roux-en-Y gastric bypass (RYGB), is associated with complications such as at-risk gastric remnant, marginal ulcers, internal hernias, and dumping syndrome. ${ }^{6}$ Laparoscopic sleeve gastrectomy with loop duodenojejunal bypass (SG LDJB) is a novel bariatric surgery found effective in terms of weight loss and diabetes remission. ${ }^{7}$ A "long common channel" reduces the risk of malabsorption. Technically, it is a loop modification of BPD DS but with more proximal anastomosis to minimize malabsorption. Literature is not available on the factors predicting weight loss response after SG LDJB. This study aimed to analyze weight loss response and various factors predicting weight loss outcomes after SG LDJB.

\section{METHODS}

This was a retrospective study carried out in 126 patients between May 2013 and December 2017 conducted at Dr. Amar Bariatric \& Metabolic Center, Hyderabad, India. Patients who underwent SG LDJB surgery for obesity were included in the study. Our study complied with the ethical principles for medical research involving human subjects in accordance with the Declaration of Helsinki. ${ }^{8}$ Institutional Review Board of Ethical Research approved this study (IRB No. ABMC/AH/2020/002) and written informed consent was obtained from all patients included in this study. All clinical and follow-up data were documented in a master data sheet. After performing sleeve gastrectomy (SG), the first part of the duodenum was disconnected and anastomosed to the jejunum, 200 to $250 \mathrm{~cm}$ distal to the duodenojejunal flexure (Fig. 1).

This study analyzed parameters including preoperative body mass index (BMI), weight, excess BMI, and excess weight (based on a BMI reference point of $25 \mathrm{~kg} / \mathrm{m}^{2}$ ) and calculated percentage excess weight loss (\%EWL), percentage excess BMI loss (\%EBMIL), percentage total weight loss (\%TWL) and percentage total BMI loss (\%TBMIL) at 1-year and 3-year follow-up intervals. For example, $\%$ EWL at 1 -year $=($ weight loss at 1 year/preoperative excess weight $) \times$

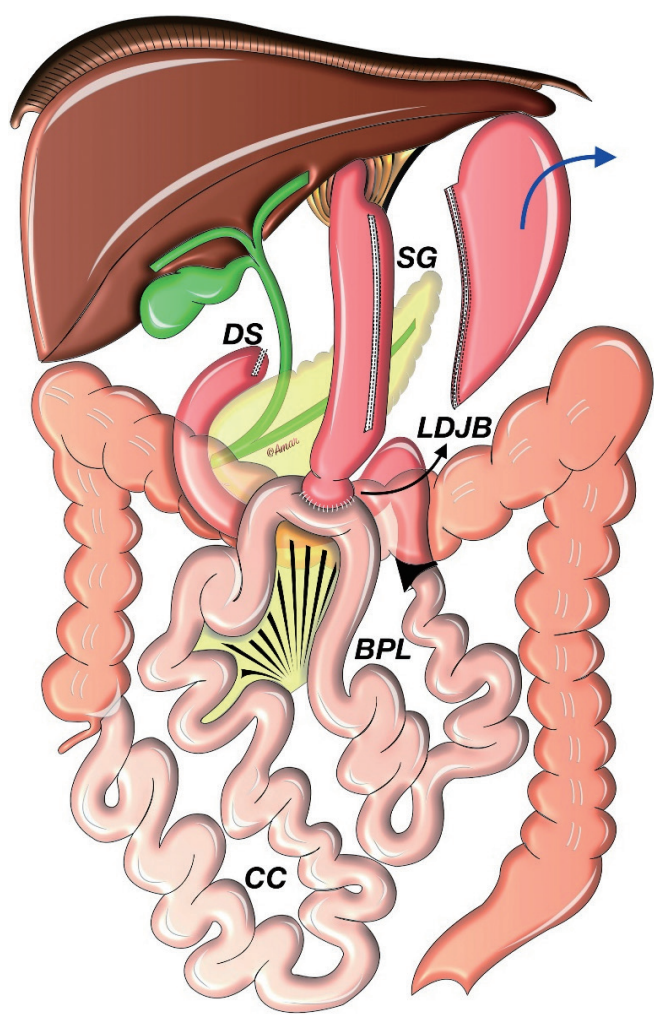

Figure 1. Pictorial diagram of "sleeve gastrectomy with loop duodenojejunal bypass": sleeve gastrectomy (SG), duodenal stump (DS), loop duodenojejunal bypass (LDJB), biliopancreatic limb (BPL), and common channel (CC).

100 and \%EBMIL at 1 year $=(\mathrm{BMI}$ loss at 1 year/preoperative excess BMI $) \times 100$. Similarly, \%TWL at 1 year $=($ weight loss at 1 year $/$ preoperative weight $) \times 100$ and $\% \mathrm{TBMIL}$ at 1 -year $=(\mathrm{BMI}$ loss at 1 year/preoperative BMI) $\times 100$. Since $\% E W L$ and $\% E B M I L$ (and \%TWL and \%TBMIL) calculations yielded exactly the same values, both were used interchangeably. A \%EWL (\%EBMIL) above 100 did not indicate that the patient lost more than $100 \%$ of excess fat; it indicated that the patient's BMI ended up below $25 \mathrm{~kg} / \mathrm{m}^{2}$.

The objective of this study was to analyze the weight loss efficacy of SG LDJB and to study the effect of age, sex, preoperative BMI, weight, excess weight, and diabetes status on \%EWL and \%TWL at the 1-year and 3-year follow-up. Weight loss parameters at the 1-year and 3-year follow-up after surgery were collected and analyzed with respect to age ( $<50$ years vs. $\geq 50$ years), sex (male vs. female), preoperative BMI $\left(<40 \mathrm{~kg} / \mathrm{m}^{2}\right.$ vs. $\left.\geq 40 \mathrm{~kg} / \mathrm{m}^{2}\right)$, and preoperative diabetes status (diabetes vs. no diabetes). Simple linear regression analysis was performed to determine the impact of various factors on \%EWL and \%TWL at the 1-year and 3-year follow-up after sur- 
gery. These factors were further analyzed by multiple regression analysis. Since the majority of patients had a BMI $<50 \mathrm{~kg} / \mathrm{m}^{2}$, a $\% \mathrm{EWL} \geq 50 \%$ was considered a successful weight loss outcome. Total weight loss (\%TWL) $\geq 25 \%$ was alternatively used to define successful weight loss outcome. Those who fell below these reference points were considered to have inadequate weight loss. Those who achieved successful weight loss at the 1-year follow-up but fell below these reference points at the 3-year follow-up were considered to have weight regain. Patients who regained weight were included with those patients who had inadequate weight loss at the 3-year follow-up. Binary logistic regression was conducted to analyze the impact of various factors on the success rate after surgery.

For this study, diabetes was considered under remission when glycosylated hemoglobin $\left(\mathrm{HbAl}_{\mathrm{C}}\right)$ was $<6.5 \%$ with a complete stoppage of insulin or oral hypoglycemic agents. ${ }^{9}$ Statistical analysis was performed using IBM SPSS version 23 (IBM Corp., Armonk, NY, USA). All $P$-values $<0.05$ in various statistical tests were considered statistically significant.

\section{RESULTS}

Between May 2013 and December 2017, SG LDJB was performed on 126 individuals suffering from obesity. Out of 126 patients, 91 were males and 35 were females. Demographic data and perioperative parameters are depicted in Table 1 . The mean BMI and weight at different time intervals are depicted in Tables 1 and 2 . The mean preoperative excess weight was $44.88 \pm 15.22 \mathrm{~kg}$ and mean excess BMI was $15.92 \pm 5.74 \mathrm{~kg} / \mathrm{m}^{2}$ (based on the BMI reference point of $\left.25 \mathrm{~kg} / \mathrm{m}^{2}\right)$. In this study, 78 of 126 patients $(61.90 \%)$ were suffering from diabetes.

BMI loss, weight loss, \%EWL, and \%TWL at the 1-year and 3-year follow-up intervals are shown in Table 2. Overall BMI loss and weight loss were significant at the 1-year and 3-year follow-up intervals compared to preoperative BMI and weight, respectively. There was an increase in BMI and weight from 1-year to 3-year follow-up, which was statistically significant.

Variances in \%EWL and \%TWL in the different groups are summarized in Table 2. Simple linear regression and multiple regression analyses at the 1-year and 3-year follow-up intervals are shown in Table 3. There was no significant difference in \%EWL and \%TWL
Table 1. Demographic and perioperative data

\begin{tabular}{lc}
\hline Variable & Value $(\mathrm{n}=126)$ \\
\hline Age $(\mathrm{yr})$ & $40.79 \pm 10.82$ \\
Age $(<50: \geq 50, \mathrm{yr})$ & $98: 28(77.78: 22.22)$ \\
Sex $(\mathrm{male}: f e m a l e)$ & $91: 35(72.22: 27.78)$ \\
$\mathrm{BMl}\left(\mathrm{kg} / \mathrm{m}^{2}\right)$ & $40.92 \pm 5.74$ \\
$\mathrm{BMl}\left(<40: \geq 40, \mathrm{~kg} / \mathrm{m}^{2}\right)$ & $60: 66(47.62: 52.38)$ \\
Weight $(\mathrm{kg})$ & $116.26 \pm 15.96$ \\
Diabetes:no diabetes & $78: 48(61.90: 38.10)$ \\
Duration of diabetes $(\mathrm{yr})$ & $4.85 \pm 4.38$ \\
Duration of surgery $(\mathrm{min})$ & $188.65 \pm 65.71$ \\
Intraoperative blood loss (mL) & $36.4 \pm 22.26$ \\
Major perioperative complications* & $2(1.59)$ \\
\hline
\end{tabular}

Values are presented as mean \pm standard deviation or number $(\%)$.

*Leakage from upper end of sleeve requiring laparoscopic drainage, and portal vein thrombosis requiring long-term anticoagulation; both cases resolved without complications.

$\mathrm{BMl}$, body mass index.

between patients aged $<50$ years vs. $\geq 50$ years at the 1 -year and 3 -year follow-up. Simple linear regression analysis showed that there was no significant correlation between age and \%EWL. Age was found to be inversely proportional to \%TWL at the 1-year follow-up, but not at the 3-year follow-up. Multiple regression analysis showed that patient age was not an independent predictor of weight loss outcomes. \%EWL was higher in males vs. females but was significant only at the 1-year follow-up. There was no significant difference in \%TWL between males and females. Multiple regression analysis showed that sex was not an independent predictor of weight loss outcomes (Table 3 ).

$\%$ EWL was higher in patients with a preoperative BMI $<40 \mathrm{~kg} / \mathrm{m}^{2}$ vs. $\geq 40 \mathrm{~kg} / \mathrm{m}^{2}$. However, $\% \mathrm{TWL}$ was higher when preoperative BMI was $\geq 40 \mathrm{~kg} / \mathrm{m}^{2}$ vs. $<40 \mathrm{~kg} / \mathrm{m}^{2}$. Simple linear regression showed that preoperative BMI, weight, and excess weight negatively predicted \%EWL and positively predicted \%TWL. Multiple regression analysis showed that these factors were independent predictors of \%EWL and \%TWL at the 1-year follow-up, while preoperative weight and excess weight were also independent predictors of \%EWL at the 3-year follow-up (Table 3). \%EWL was higher in non-diabetic patients vs. diabetic patients but was significant only at the 3-year follow-up. \%TWL was significantly higher in non-diabetic patients compared to diabetic patients at the 1-year and 3-year follow-up. Multiple regression analysis confirmed these findings.

Weight loss success rate and inadequate weight loss at the 1-year 
Table 2. Weight loss parameters

\begin{tabular}{|c|c|c|c|c|c|c|}
\hline \multirow{2}{*}{ Variable } & \multicolumn{3}{|c|}{ 1-Year follow-up } & \multicolumn{3}{|c|}{ 3-Year follow-up } \\
\hline & $\mathrm{n}$ & Mean \pm SD & $P$ & $n$ & Mean \pm SD & $P$ \\
\hline Weight & 104 & $75.44 \pm 11.08$ & $<0.001^{*}$ & 85 & $79.18 \pm 12.12$ & $<0.001^{\dagger}$ \\
\hline $\mathrm{BMI}\left(\mathrm{kg} / \mathrm{m}^{2}\right)$ & 104 & $26.78 \pm 3.74$ & $<0.001^{*}$ & 85 & $28.38 \pm 4.16$ & $<0.001^{\dagger}$ \\
\hline Weight Loss (kg) & 104 & $40.45 \pm 10.13$ & - & 85 & $35.02 \pm 11.25$ & $<0.001^{\dagger}$ \\
\hline BMI loss $\left(\mathrm{kg} / \mathrm{m}^{2}\right)$ & 104 & $14.39 \pm 3.66$ & - & 85 & $12.6 \pm 4.09$ & $<0.001^{\dagger}$ \\
\hline \%Excess weight loss & 104 & $95.77 \pm 25.26$ & - & 85 & $83.84 \pm 24.65$ & $<0.001^{\dagger}$ \\
\hline \multicolumn{7}{|l|}{ Subgroup } \\
\hline Age $(<50$ yr $)$ & 77 & $96.35 \pm 25.38$ & $0.694^{\ddagger}$ & 60 & $82.88 \pm 23.06$ & $0.580^{\ddagger}$ \\
\hline Age ( $\geq 50 \mathrm{yr})$ & 27 & $94.12 \pm 25.3$ & & 25 & $86.15 \pm 28.5$ & \\
\hline Male & 75 & $98.88 \pm 27.35$ & $0.014^{\ddagger}$ & 60 & $85.11 \pm 24.78$ & $0.465^{\ddagger}$ \\
\hline Female & 29 & $87.74 \pm 16.68$ & & 25 & $80.79 \pm 24.58$ & \\
\hline $\mathrm{BMI}\left(<40 \mathrm{~kg} / \mathrm{m}^{2}\right)$ & 49 & $111.97 \pm 24.82$ & $<0.001^{\ddagger}$ & 43 & $95.33 \pm 26.22$ & $<0.001^{\ddagger}$ \\
\hline $\mathrm{BMI}\left(\geq 40 \mathrm{~kg} / \mathrm{m}^{2}\right)$ & 55 & $81.35 \pm 14.82$ & & 42 & $72.08 \pm 16.14$ & \\
\hline Diabetes & 77 & $95.26 \pm 26.65$ & $0.696^{\ddagger}$ & 73 & $80.81 \pm 22.67$ & $0.005^{\ddagger}$ \\
\hline No diabetes & 27 & $97.25 \pm 21.19$ & & 12 & $102.28 \pm 29.09$ & \\
\hline \%Total weight loss & 104 & $34.64 \pm 5.88$ & - & 85 & $30.32 \pm 7.6$ & $<0.001^{\dagger}$ \\
\hline \multicolumn{7}{|l|}{ Subgroup } \\
\hline Age (<50 yr) & 77 & $35.28 \pm 5.63$ & $0.064^{\ddagger}$ & 60 & $30.14 \pm 8.06$ & $0.739^{\ddagger}$ \\
\hline Age ( $\geq 50 \mathrm{yr}$ ) & 27 & $32.84 \pm 6.32$ & & 25 & $30.75 \pm 6.49$ & \\
\hline Male & 75 & $34.07 \pm 5.93$ & $0.109^{\ddagger}$ & 60 & $29.32 \pm 7.57$ & $0.059^{\ddagger}$ \\
\hline Female & 29 & $36.13 \pm 5.57$ & & 25 & $32.73 \pm 7.26$ & \\
\hline $\mathrm{BMI}\left(<40 \mathrm{~kg} / \mathrm{m}^{2}\right)$ & 49 & $32.7 \pm 5.4$ & $0.001^{\ddagger}$ & 43 & $28.2 \pm 8.15$ & $0.009^{\ddagger}$ \\
\hline $\mathrm{BMI}\left(\geq 40 \mathrm{~kg} / \mathrm{m}^{2}\right)$ & 55 & $36.37 \pm 5.79$ & & 42 & $32.49 \pm 6.39$ & \\
\hline Diabetes & 77 & $33.64 \pm 5.5$ & $0.003^{\ddagger}$ & 73 & $29.05 \pm 6.85$ & $<0.001^{\ddagger}$ \\
\hline No diabetes & 27 & $37.49 \pm 6.1$ & & 12 & $38.07 \pm 7.62$ & \\
\hline
\end{tabular}

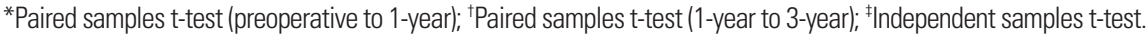

$\mathrm{SD}$, standard deviation; BMl, body mass index.

Table 3. Significance on regression analysis

\begin{tabular}{|c|c|c|c|c|c|c|c|c|c|c|c|c|}
\hline \multirow{3}{*}{$\begin{array}{l}\text { Significance* } \\
\text { Predictor }\end{array}$} & \multicolumn{4}{|c|}{ Simple linear regression } & \multicolumn{4}{|c|}{ Multiple regression analysis } & \multicolumn{4}{|c|}{ Binary logistic regression } \\
\hline & \multicolumn{2}{|c|}{$\% E W L$} & \multicolumn{2}{|c|}{$\%$ TWL } & \multicolumn{2}{|c|}{$\% E W L$} & \multicolumn{2}{|c|}{$\%$ TWL } & \multicolumn{2}{|c|}{$\% E W L \geq 50$} & \multicolumn{2}{|c|}{$\% T W L \geq 25$} \\
\hline & 1 Year & 3 Years & 1 Year & 3 Years & 1 Year & 3 Years & 1 Year & 3 Years & 1 Year & 3 Years & 1 Year & 3 Years \\
\hline Age & 0.456 & 0.713 & 0.013 & 0.555 & 0.161 & 0.221 & 0.114 & 0.581 & 0.997 & 0.551 & 0.159 & 0.541 \\
\hline $\operatorname{Sex}^{\dagger}$ & - & - & - & - & 0.973 & 0.763 & 0.566 & 0.672 & 0.999 & 0.998 & 0.784 & 0.863 \\
\hline Preoperative BMI & $<0.001$ & $<0.001$ & $<0.001$ & $<0.001$ & $<0.001$ & 0.121 & 0.015 & 0.386 & 0.997 & 0.215 & 0.064 & 0.262 \\
\hline Preoperative weight & $<0.001$ & $<0.001$ & 0.001 & 0.004 & $<0.001$ & 0.049 & 0.045 & 0.820 & 0.996 & 0.410 & 0.050 & 0.337 \\
\hline Preoperative EW & $<0.001$ & $<0.001$ & $<0.001$ & $<0.001$ & $<0.001$ & 0.032 & 0.031 & 0.662 & 0.996 & 0.255 & 0.062 & 0.334 \\
\hline Diabetes status ${ }^{\ddagger}$ & - & - & - & - & 0.428 & $<0.001$ & 0.030 & $<0.001$ & 0.997 & 0.482 & 0.658 & 0.208 \\
\hline
\end{tabular}

Variables included in multiple regression analysis: age, sex, preoperative BMI, preoperative weight, preoperative EW, diabetes status.

${ }^{*} P$-value in various tests; ${ }^{\dagger}$ Male vs. female; ${ }^{\prime}$ Diabetes vs. no diabetes.

\%EWL, percentage excess weight loss (with $25 \mathrm{BMI}$ reference point); \%TWL, percentage total weight loss; BMI, body mass index; EW, excess weight.

and 3-year follow-up are documented in Table 4. Those patients who regained weight at the 3-year follow-up after achieving successful weight loss at the 1-year follow-up are also documented in Table 4. Binary logistic regression showed that none of the preop- erative factors influenced success rate (Table 3 ).

Percentage of diabetes remission, improvement with $\mathrm{HbA1} 1_{\mathrm{C}}$ $<6.5 \%$ taken as cut-off, use of insulin and oral hypoglycemic agents are depicted in Table 5. None of the diabetic patients had di- 
Table 4. Weight loss success rate

\begin{tabular}{lcc}
\hline Parameter & 1 Year & 3 Years \\
\hline Based on \%EWL $\geq 50$ reference point & & \\
Successful weight loss (\%EWL $\geq 50)$ & $99.04(103 / 104)$ & $96.47(82 / 85)$ \\
Inadequate weight loss (\%EWL <50) & $0.96(1 / 104)$ & $3.53(3 / 85)$ \\
Weight regain at the 3-year follow-up* & - & $2.38(2 / 84)^{\dagger}$ \\
Based on \%TWL $\geq 25 \%$ reference point & & \\
Successful weight loss (\%TWL $\geq 25)$ & $91.35(95 / 104)$ & $75.29(64 / 85)$ \\
Inadequate weight loss (\%TWL <25) & $8.65(9 / 104)$ & $24.71(21 / 85)$ \\
Weight regain at the 3-year follow-up & - & $18.18(14 / 77)^{\ddagger}$ \\
\hline
\end{tabular}

Values are presented as percent (number).

*Included in corresponding inadequate weight loss subgroup; ${ }^{\dagger}$ Out of 103 patients who had successful weight loss (\%EWL $\geq 50$ ) at 1 year, 84 were available at the 3 -year follow-up; ${ }^{\ddagger}$ Out of 95 patients who had successful weight loss (\%TWL $\geq 25$ ) at 1 year, 77 were available at the 3-year follow-up.

\%EWL, percentage excess weight loss; \%TWL, percentage total weight loss.

abetes deterioration. Even though some patients who achieved remission had recurrence of diabetes later, they were still in the diabetes improvement category when compared to their preoperative diabetes status. None of our patients developed protein energy malnutrition after SG LDJB. None of the patients required revision in the form of adjusting the length of the biliopancreatic limb for weight regain or nutritional complications.

\section{DISCUSSION}

Bariatric surgery is an effective remedy to treat obesity. Weight loss after bariatric surgery is mainly due to physiological and hormonal changes including a reduction in ghrelin, anti-incretins, and an increase in distal ileal hormones such as glucagon-like peptide 1 and peptide YY, which reset fat mass to a lower limit. The same physiological changes are responsible for diabetes remission. ${ }^{10}$

SG LDJB is a novel metabolic surgery carried out with the objective to reduce malabsorption while maintaining surgery efficacy. ${ }^{11}$ Endoscopic surveillance of the gastric sleeve is possible after this surgery and there is no at-risk gastric remnant. Anastomosing in a loop fashion rather than in the Roux-en-Y fashion reduces the number of anastomoses and mesenteric gaps, which probably translates into a reduced risk of internal hernias. ${ }^{11}$

In our study, the mean \%EWL and \%TWL were greatest at the 1-year follow-up. These were relatively lower at the 3-year followup, but still \%EWL was $\geq 50 \%$ in $>95 \%$ of patients and $\% \mathrm{TWL}$ was $\geq 25 \%$ in $>75 \%$ of patients, indicating that the weight loss re-
Table 5. Diabetes parameters

\begin{tabular}{lccc}
\hline Parameter & Preoperative & 1 Year & 3 Years \\
\hline Response with HbA1c <6.5\% cut-off & & & \\
Remission (\%) & - & $90.91(70 / 77)$ & $90.41(66 / 73)$ \\
Improvement (\%) & - & $9.09(07 / 77)$ & $9.59(07 / 73)$ \\
Recurrence (\%) & - & - & $4.48^{*}(03 / 67)^{\dagger}$ \\
Usage of insulin & $30.77(24 / 78)$ & $0(0 / 77)$ & $0(0 / 73)$ \\
Usage of oral hypoglycemic agents & $84.62(66 / 78)$ & $6.49(5 / 77)$ & $6.85(5 / 73)$ \\
\hline
\end{tabular}

Values are presented as percent (number).

*Patients with recurrence are in the improvement category compared to preoperative status; ${ }^{\top}$ Sixty-seven of 70 patients who achieved remission at 1 year were available at the 3-year follow-up.

$\mathrm{HbA1}$ c, glycosylated hemoglobin.

sponse was durable with SG LDJB. Similarly, mean BMI loss and mean weight loss were greatest at the 1-year follow-up. In a study by Maciejewski et al., ${ }^{12}$ patients who underwent RYGB, laparoscopic adjustable gastric banding (LAGB), or SG lost 30.9\%, 13\%, and 23.4\% of their baseline weight, respectively, at the 1-year follow-up. At 4 years, patients who underwent RYGB, LAGB, or SG lost 27.5\%, 10.65 , and $17.8 \%$ of their base-line weight, respectively. ${ }^{12}$

In our study, the proportion of inadequate weight loss with $\% \mathrm{EWL}<50 \%$ was $<1 \%$ at the 1 -year and $<5 \%$ at the 3 -year follow-up. When $\% \mathrm{TWL}<25 \%$ was taken into consideration, inadequate weight loss was $<10 \%$ at the 1 -year and $<25 \%$ at the 3 -year follow-up. This indicated that even though there was weight regain and an increase in BMI after 1-year, overall weight loss was maintained over a period of time after SG LDJB. Inadequate weight loss and weight regain are mainly related to a failure to maintain postoperative lifestyle modifications. Lifestyle modifications, regular follow-ups with the surgeon and dietician, and attending support group meetings may help patients to maintain post-surgery weight loss.

According to Ballantyne ${ }^{2}$, younger patients had greater \%EWL than older patients following RYGB and vertical banded gastroplasty bariatric procedures. In our study, age did not alter \%EWL but younger patients had better \%TWL at the 1-year follow-up. However, patient age and sex were not found to be independent predictors of weight loss outcomes after SG LDJB.

In our study, it was found that preoperative BMI negatively predicted \%EWL, indicating that lower BMI patients had higher \%EWL. However, preoperative BMI positively predicted \%TWL, indicating that higher BMI patients had higher \%TWL. Patients with mild 
and severe obesity $\left(\mathrm{BMI}<40 \mathrm{~kg} / \mathrm{m}^{2}\right)$ have better \%EWL after this surgery compared to those with morbid obesity $\left(\mathrm{BMI} \geq 40 \mathrm{~kg} / \mathrm{m}^{2}\right)$. These results are similar to the study by Ochner et al., ${ }^{13}$ which showed that it was preferable to perform bariatric surgery when obesity was still in the initial stages as achieving a significant reduction in weight was easier in early stages of obesity. In this study, patients with a preoperative BMI of 35 to $49.9 \mathrm{~kg} / \mathrm{m}^{2}$ continued to lose weight, while individuals with a BMI $\geq 50 \mathrm{~kg} / \mathrm{m}^{2}$ regained significant weight, from 1 to 3 years after surgery. ${ }^{13}$

Our study showed that the presence of diabetes negatively predicted \%EWL at the 3-year follow-up and \%TWL at 1 and 3 years, indicating that non-diabetic patients responded well after SG LDJB. Sillén and Andersson ${ }^{14}$ showed that the presence of diabetes reduces \%EWL after RYGB surgery.

Our study showed that the success rate after surgery was high at the 1-year follow-up and remained high even at the 3-year followup. Even though some preoperative factors predicted a weight loss response, they failed to influence success rate. It is probable that the high success rate after SG LDJB might have masked the influence of various factors. Diabetes remission reached a maximum at the 1-year follow-up in our patients with $>90 \%$ patients with $\mathrm{HbAl}_{\mathrm{C}}$ levels $<6.5 \%$, even in the absence of taking any medications at the 1-year follow-up. This study showed that patients achieved significant glycemic control after SG LDJB without using any medications. Even at the 3-year follow-up, remission was >90\%, indicating that diabetes remission was durable after SG LDJB.

Significant weight loss after SG LDJB was perhaps more related to physiological and hormonal changes. The longer biliopancreatic limb may also contribute to an increase in weight loss. ${ }^{15}$ Increasing the length of the biliopancreatic limb can increase protein energy malnutrition. In most of our SG LDJB patients, biliopancreatic limb length was $250 \mathrm{~cm}$ and protein energy malnutrition was not observed. Preservation of the pylorus in SG LDJB probably played a role in preventing protein energy malnutrition. Since the pylorus controls gastric emptying, a longer length of the intestine could be bypassed without adverse malabsorptive outcomes. ${ }^{16}$ Detailed discussion of nutritional deficiencies after SG LDJB was beyond the scope of this article.

One drawback of this study was that there might have been bias while selecting different bariatric procedures for obesity and diabe- tes. While SG is preferred in teenagers with mild obesity and females planning for future pregnancy, SG LDJB is preferred in all other patients with $\mathrm{BMI}<50 \mathrm{~kg} / \mathrm{m}^{2}$ and single anastomosis duodenoileal bypass with sleeve was preferred in those with a BMI $\geq 50 \mathrm{~kg} / \mathrm{m}^{2}$. SG LDJB was preferentially offered to diabetic patients with $\mathrm{BMI}<50 \mathrm{~kg} / \mathrm{m}^{2}$. Another drawback with this surgery was that the second part of the duodenum was excluded, obviating endoscopic access to the biliary tract. Furthermore, SG LDJB surgery is technically complex with a long learning curve. The results indicate that SG LDJB appears to be a promising surgery with durable \%EWL and \%TWL. Long-term data are required to evaluate the exact efficacy of this novel surgery.

The study showed that SG LDJB is a physiological and efficacious bariatric procedure in terms of weight loss response. Preoperative BMI, weight, excess weight, and presence of diabetes significantly predicted weight loss outcomes after SG LDJB.

\section{CONFLICTS OF INTEREST}

The authors declare no conflict of interest.

\section{AUTHOR CONTRIBUTIONS}

Study concept and design: $\mathrm{AV}$; acquisition of data: $\mathrm{AV}$; analysis and interpretation of data: all authors; drafting of the manuscript: $\mathrm{AV}$; critical revision of the manuscript: all authors; statistical analysis: all authors; administrative, technical, or material support: $\mathrm{CK}_{\text {; }}$ and study supervision: AV.

\section{REFERENCES}

1. Szczuko M, Komorniak N, Hoffmann M, Walczak J, Jaroszek A, Kowalewski B, et al. Body weight reduction and biochemical parameters of the patients after RYGB and SG bariatric procedures in 12-month observation. Obes Surg 2017;27:940-7.

2. Ballantyne GH. Measuring outcomes following bariatric surgery: weight loss parameters, improvement in co-morbid conditions, change in quality of life and patient satisfaction. Obes Surg 2003;13:954-64.

3. Capristo E, Panunzi S, De Gaetano A, Raffaelli M, Guidone C, 
Iaconelli A, et al. Intensive lifestyle modifications with or without liraglutide $3 \mathrm{mg}$ vs. sleeve gastrectomy: a three-arm nonrandomised, controlled, pilot study. Diabetes Metab 2018;44: 235-42.

4. Copăescu C. Laparoscopic biliopancreatic diversion with duodenal switch: the most effective operation for type 2 diabetes mellitus. How I do it? Chirurgia (Bucur) 2018;113:704-11.

5. Homan J, Betzel B, Aarts EO, Dogan K, van Laarhoven KJ, Janssen IM, et al. Vitamin and mineral deficiencies after biliopancreatic diversion and biliopancreatic diversion with duodenal switch: the rule rather than the exception. Obes Surg 2015; 25:1626-32.

6. Huang CK, Tai CM, Chang PC, Malapan K, Tsai CC, Yolsuriyanwong K. Loop duodenojejunal bypass with sleeve gastrectomy: comparative study with Roux-en-Y gastric bypass in type 2 diabetic patients with a BMI $<35 \mathrm{~kg} / \mathrm{m} 2$, first year results. Obes Surg 2016;26:2291-301.

7. Nor Hanipah Z, Hsin MC, Liu CC, Huang CK. Laparoscopic loop duodenaljejunal bypass with sleeve gastrectomy in type 2 diabetic patients. Surg Obes Relat Dis 2019;15:696-702.

8. World Medical Association. World Medical Association Declaration of Helsinki: ethical principles for medical research involving human subjects. JAMA 2013;310:2191-4.

9. Lean ME, Leslie WS, Barnes AC, Brosnahan N, Thom G, McCombie L, et al. Primary care-led weight management for re- mission of type 2 diabetes (DiRECT): an open-label, clusterrandomised trial. Lancet 2018;391:541-51.

10. Kim KS, Sandoval DA. Endocrine function after bariatric surgery. Compr Physiol 2017;7:783-98.

11. Huang CK, Goel R, Tai CM, Yen YC, Gohil VD, Chen XY. Novel metabolic surgery for type II diabetes mellitus: loop duodenojejunal bypass with sleeve gastrectomy. Surg Laparosc Endosc Percutan Tech 2013;23:481-5.

12. Maciejewski ML, Arterburn DE, Van Scoyoc L, Smith VA, Yancy WS Jr, Weidenbacher HJ, et al. Bariatric surgery and long-term durability of weight loss. JAMA Surg 2016;151: 1046-55.

13. Ochner CN, Jochner MC, Caruso EA, Teixeira J, Xavier PiSunyer F. Effect of preoperative body mass index on weight loss after obesity surgery. Surg Obes Relat Dis 2013;9:423-7.

14. Sillén L, Andersson E. Patient factors predicting weight loss after Roux-en-Y gastric bypass. J Obes 2017;2017:3278751.

15. Jia D, Tan H, Faramand A, Fang F. One anastomosis gastric bypass versus Roux-en-Y gastric bypass for obesity: a systematic review and meta-analysis of randomized clinical trials. Obes Surg 2020;30:1211-8.

16. Roslin M, Tugertimur B, Zarabi S, Cottam D. Is there a better design for a bariatric procedure? The case for a single anastomosis duodenal switch. Obes Surg 2018;28:4077-86. 